Research Article

\title{
A Novel Method for Laser Peak Detection with Subpixel Accuracy for the Rail Corrugation Measurement
}

\author{
Mehran Torabi, S. Mohammad Mousavi G $\mathbb{D}$, and Davood Younesian $(\mathbb{C}$ \\ School of Railway Engineering, Iran University of Science and Technology, Tehran, Iran \\ Correspondence should be addressed to Davood Younesian; younesian@iust.ac.ir
}

Received 20 December 2020; Accepted 3 September 2021; Published 1 October 2021

Academic Editor: Qiang Wu

Copyright (@ 2021 Mehran Torabi et al. This is an open access article distributed under the Creative Commons Attribution License, which permits unrestricted use, distribution, and reproduction in any medium, provided the original work is properly cited.

\begin{abstract}
In this article, a new wavelet-based laser peak detection algorithm is proposed having subpixel accuracy. The algorithm provides an accurate and rapid measurement platform for the rail surface corrugation with no need to any image noise elimination. The proposed rail Corrugation Measurement System (CMS) is based on the laser triangulation principle, and the accuracy of such system is mainly affected by the laser peak detection in the captured image. The intensity of each row or column of the image is taken as a 1-D discrete signal. Intensity distribution of a laser stripe in this signal follows a Gaussian pattern contaminated by the white noise. Against usual peak detection algorithms with need to prenoise-filtering process, the proposed method based on the wavelet transform is able to perform these tasks efficiently and robustly. Present wavelet-based methods for the peak detection are at pixel level, but for achieving high accuracy subpixel detection is proposed. Experiments show that the capability of the proposed method for laser peak detection is more accurate and faster than the filter-based methods, especially for low $\mathrm{S} / \mathrm{N}$ ratios. Also, this technique can be utilized for any application in laser peak detection with subpixel accuracy. A prototype system based on the proposed method for the rail corrugation measurement has been designed and manufactured. Results of the rail corrugation measurement guarantee capability of the proposed methodology for accurate measurement of the rail corrugation and its potential for industrial application.
\end{abstract}

\section{Introduction}

Rail corrugation as a quasisinusoidal irregularity along the longitudinal direction of the rail occurs on the top of the rail due to wear when a railway vehicle runs over it. It causes airborne noise or ground-borne vibration which decreases the travel safety and level of the environment-friendliness, especially in subway or high speed tracks [1-3]. Thus, investigation and monitoring rail health through measurement of its longitudinal profile, permanently and regularly, is a necessary task for railways.

There are several methods for measuring rail corrugation [4]. They can be divided into three main categories: (i) chord-based measuring methods, (ii) low speed accelerometer-based techniques, and (iii) axle box accelerometer systems. Chord-based method is the first technique used to measure the longitudinal profile of rails and is still probably the most widely used method. Measurements are made from a chord with the ends on the rail. The reference of the measurement system moves with vehicle and a transducer on the chord measures the distance to the rail surface. The disadvantage of this method is the dependency of the measurements to corrugation wavelength.

In accelerometer-based profile measurement methods, the main advantage is that the measuring system has an inertial reference that does not depend on the path. A low speed accelerometer system for measuring the longitudinal profile of a rail has been described in [5]. At frequencies much lower than the accelerometer resonance frequency, the measured profile is the same as the input profile. Therefore, to have an ideal measurement, the measurement speed must be very low, and it is difficult to use such a system in a railway vehicle, especially when traveling at line speeds. Another challenge to use an accelerometer based method is its sensitivity to the vibration of the rail. For high speed rail profile measurement, axle box accelerometer methods have been developed [6-8]. The acceleration of the wheel set as a rigid body is measured. In fact, measuring acceleration is 
a direct technique to measure dynamic contact force and not for profile measurement. In addition, the measurement system is not an ideal system, since the results depend not only on the speed of the vehicle but also on the characteristics of the track.

Recently, a new approach based on the principle of laser triangulation has been proposed in [9] that its results are independent of the track quality; however, its accuracy depends on the detection of the peak of the laser stripe in the image captured by the system. Thus, accurate detection of laser peak has an important role in accurate measurement of 2-D rail profile [10]. In general, the laser peak detection methods utilize a special color channel (such as red channel) for an RGB image or grayscale channel for a grayscale image (that originally obtained from a camera or by converting an RGB image to a grayscale image). The value of a pixel in each row or column of an image represents the intensity of light; so, the intensity along that row or column is considered as a 1-D signal that makes the laser peak detection problem as signal peak detection. The easiest way for signal peak detection is the maximum value method. The profile is achieved only by locating the pixel right at the maximum amount of intensity. Due to the accuracy of the detection at the pixel level, two challenges may appear: low accuracy and noise sensitiveness [11].

According to the demand of a laser camera measurement system for higher measurement accuracy, the subpixel peak detection methods have received more attention. There are several well-known methods for achieving subpixel accuracy that consist of 1-Gaussian approximation method, 2-center of the mass method, 3-Blais and Rioux detectors, 4-parabolic estimator, 5-linear interpolation method, and 6-zero crossing of the first derivative method (FIR-finite impulse response filter approach) [12]. The efficiency of the first five methods has been discussed in [13], and the last one has been described in $[14,15]$. The center of mass or gray-gravity method is an efficient method, which is simple and easy to use [16-19]. Due to the sensitivity of the addressed techniques to noise, results of measuring profiles are very much affected by it. To reduce the effect of the input signal noise on the measurement results, it is necessary to eliminate the noise through a low-pass filter or to augment the peak by convolving it to a Gaussian kernel (or crosscorrelation). Accordingly, there are two approaches to complete the process: 1-low-pass filtering and 2-peak augmentation through convolution (or crosscorrelation). In low-pass filtering methods, an appropriate filter is designed for smoothing the intensity signal. One way to determine the required filter and its coefficients is utilizing a Fourier transform. Applying Fourier transform to signal and smoothing it is a very useful technique for eliminating the noise. It should be emphasize that, in the first approach, the nature of the noise and its characteristic is the basis, while in the second approach, the shape of the signal and its similarity to a specific pattern is important. In practice, the second approach is more robust for peak detection, due to the high power of the laser peak compared to the noise.

By considering the Gaussian distribution of a laser stripe, one of the commonly used methods, which was originally designed for analyzing the medical image, is the Steger method [20] which has high robustness and accuracy in $3 \mathrm{D}$ coordinates measurement systems [21]. During the peak detection process, all the data in the input signal (pixels in the captured image) convolve with the Gaussian kernel, and to achieve subpixel accuracy, the eigenvalues and the eigenvectors of each Heissen matrix are solved [22]. This therefore requires a huge amount of calculation and also long processing time. The result relies highly on the parameters such as the standard deviation of the Gaussian pattern and the threshold of the eigenvalues [23]. By a set of fixed parameters, this method is hardly compatible with stripes with different widths and noise amplitudes.

There are other techniques based on the Gaussian correlation. Cai et al. used the principal component analysis method for estimating the normal direction, and then subpixel laser peak was calculated using the second order Taylor expansion [24]. This method is also has high computational expense. Sun et al. proposed a robust laser peak detection method which is based on the grey level moment and the smoothing spline algorithm [25]. This analysis is based on the fact that the light intensity is uniformly distributed. Meanwhile, crosssectional intensity of most laser stripes follows the Gaussian distribution [26]. In addition, some methods are through calculation of crosscorrelation [27-29]. The effect of noise on the signal peak detection can be effectively suppressed, but these methods require a large amount of computation due to crosscorrelation process.

While the above noise filtering algorithms and peak detection methods have been developed for the signal peak detection, wavelet transform analysis is able to perform both of tasks efficiently and simultaneously. Accordingly, waveletbased methods for the peak detection are considered in the analysis of spectral data $[30,31]$. The wavelet transform template matching is capable of detecting the signal peak with different latitudes and domains, but at pixel level. So, low accuracy is a consequent disadvantage.

In this paper, a wavelet-based laser peak detection algorithm proposed at subpixel accuracy. The wavelet transform is utilized for the pattern matching (especially a Gaussian pattern), and its coefficients are used for subpixel accuracy. The wavelet transform pattern matching can robustly detect the laser peak at pixel level, and for achieving the subpixel accuracy, the appropriate coefficients of the wavelet transform have been utilized. Thus, by use of the wavelet transform pattern matching and by calculating its coefficients, the peak of the laser stripe is accurately detected, and there is no need to smooth the signal in the presence of noise. The proposed method is useful for applications such as laser peak detection in the laser triangulation measurement system. Some experiments are carried out to compare the proposed method with the high accuracy techniques that first smooth the signal with a suitable filter and then detect the signal peak. Results show that the subpixel accuracy obtained through the proposed method is better than those obtained by the others. This method is presented to be utilized in an exact geometric measurement system for the rail corrugation investigation.

The rest of the paper is categorized as follows: the proposed corrugation measurement system is explained in Section II. Section III presents the laser light modeling. 
Section IV explains the nature of the noises produced in an imaging system. Section V discusses the proposed method. Section VI presents the results of the experiments. Finally, Section VII ends up with the conclusion.

\section{Proposed Corrugation Measurement System}

The proposed corrugation measurement system is a type of laser measurement system which works based on the triangulation principle. Design of a machine vision system for industrial usage has been explained in [32]. Major parameters that are important for a rail corrugation measurement system based on this principle are as follows: the corrugation wavelength and amplitude, repeatability, accuracy, reliability, cost, and the imaging speed. Moreover, environmental issues such as environment light, surface reflection, dust, temperature, and humidity should be also taken into design considerations. All of the parameters and constraints should be taken into account as well as the software and hardware requirements for the system.

2.1. System Configuration. The measurement system consists of a digital SLR camera (Canon EOS 500D, $22.3 \times 14.9 \mathrm{~mm}$ sensor size, ISO speed of 100-3200, a lens kit with $18-55 \mathrm{~mm}$ variable focal length, provides up to $4752 \times 3168$ still image resolution) for acquisition an image with shutter speed up to "4000" (1/4000th of a second), three laser emitters equipped with a suited lens to project three laser lines onto the rail surface, a basis for keeping the whole setup on a car body, and a digital processing system include HP EliteBook Folio $9470 \mathrm{M}$, which processes the image taken from the camera to extract the rail corrugation of the track. The proposed corrugation measurement system is shown in Figure 1.

Camera uses a USB interface to connect to the host laptop for processing the captured images. On the other hand, each light projected onto the rail surface is a laser line generated by a laser diode with a power of $50 \mathrm{~mW}$ and a cylindrical lens. The laser beam wavelength is about $685 \mathrm{~nm}$. Thus, using an optical filter, the imaging device is able to easily capture the laser beams reflected from the rail surface while reducing the ambient noise effects.

2.2. Measurement Principle. Corrugation as a quasisinusoidal irregularity appears on the running surface of the rails and normally has wavelength of less than one meter. The present corrugation measurement system works based on laser triangulation principle.

For the rail corrugation, the 2-D profile of the rail in longitudinal direction should be measured. CMS device should be perpendicular to the rail surface and parallel along the rail longitudinal direction for a perfect measurement. In practice, these two conditions sometimes may not be met. The measurement error due to nonperpendicularity of the CMS device as well as nonparallel laser lines along the rail longitudinal direction can be eliminated through projecting the extracted 2-D profile to the measurement plane which passes through rail longitudinal and surface normal axes.

The corrugation of the rail is measured at the head of the rail. The instrument should align mechanically on a car

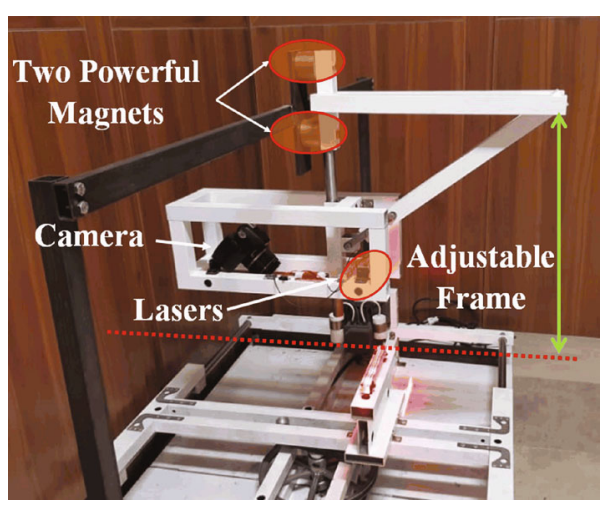

FIgURE 1: Configuration of the proposed CMS.

body. If the angle of deviation of the CMS device from the longitudinal direction of the rail is less than 10 degrees, the measurement error is less than $1.5 \%$ in corrugation wavelength. Running surface is defined as the contact area between the rail and the wheel where the wheel is most often in contact with the rail. The use of three laser planes enables the CMS to cover the entire running surface for measurement. Also, with several lasers' lines, the normal surface of the rail can be calculated. Once the surface normal is obtained, the 2-D extracted profile can be corrected.

\section{Laser Light Modeling}

A typical kind of the laser measurement system based on the triangulation principle is shown in Figure 2. This system consists of a laser and a camera to measure the geometric shape, especially $2-\mathrm{D}$ profile of an object. In order to measure a profile, the laser stripe peak needs to be determined in the image captured by the system.

The reflection of the laser light is different for each surface, depending on its optical property, specular reflection, or diffusion will happen. Dealing with a rail surface, due to its roughness (diffuse and specular combined reflection), reflection usually behaves like Figure 3 .

An image captured by the camera from the reflection of the laser light from such a surface shows that the distribution of the laser intensity in the monochrome or grayscale follows a Gaussian pattern. The pattern is illustrated in Figure 4.

For convenience, each row/column of the image can be considered as a 1-D input signal representing the light intensity along that row/column, and the laser peak is detected by identifying the maximum value of the light intensity in the input signal. Thus, by considering each row/column of the image as an input 1-D signal and by modeling the laser light distribution as a Gaussian pattern, identifying the maximum value problem converts into a template matching (Gaussian wavelet) issue. Figure 5 shows a sample of the laser stripe in a captured image with intensity distribution of 1-D input signal in a specific column.

Under controlled conditions of ambient light and laser intensity, the laser peak detection is a direct task. In industrial application especially for the railway usage, images are affected by environmental light, multiple reflections, rail 


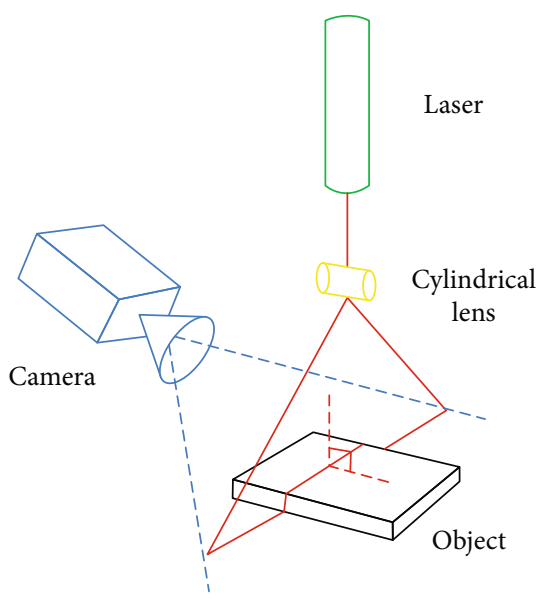

Figure 2: Schematic of a typical triangulation-based laser measurement system.

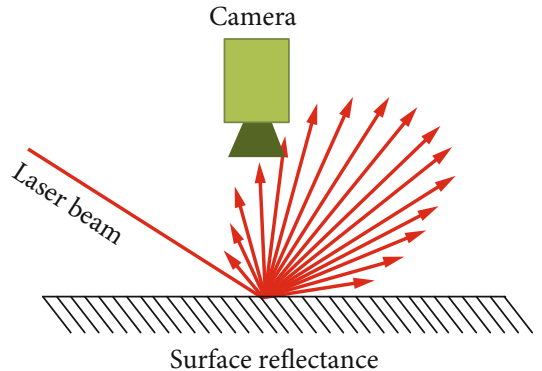

FIgURE 3: Distribution of laser light reflected from a rail surface (diffuse and specular combined reflection).

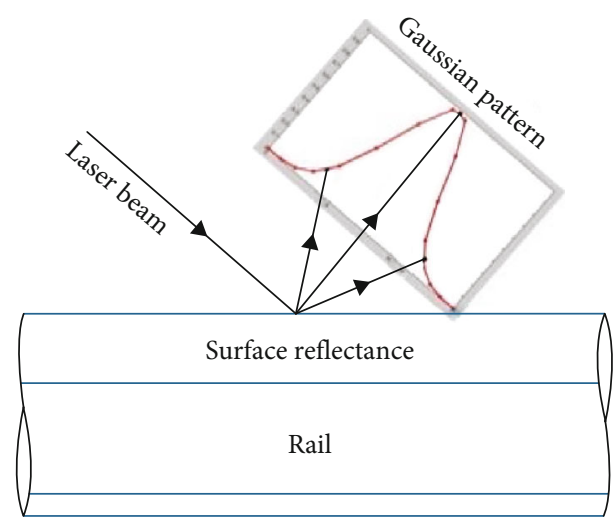

Figure 4: Distribution of laser light reflected from a rail surface.

oxidation, greasy surfaces, speckle effect, optical aberrations, and image sensor saturation.

One of the most efficient ways to reduce the influence of environmental light on the accuracy of measurement is using shroud coverage. A laser camera system based on the image processing has been developed for real-time vertical track deflection measurement [33]. In this system, the ambient light, specifically the sunlight, ruins the image of the laser lines across the rail and makes it impossible for the image processing program to distinguish the laser lines. Therefore, a shroud assembly has been utilized to shade the sunlight. Even in very sunny conditions, the shroud provides adequate shading for the laser and camera image. Also, a camera lens hood (cylindrical lens hood or petal lens hood) is useful.

In addition, one of the most important design parameters in developing a machine vision system is the dominant type of lighting, which depends on the light source and the related optics [32]. For this purpose, using the coherence characteristic of laser source with wavelength of $680 \mathrm{~nm}$, a special lens (a narrow band filter) with wavelength of $680 \mathrm{~nm}$ has been added to the camera to suppress the unrequested lights input. Also, the camera setting especially exposure time has been changed to decrease the influence of ambient light on the measurement accuracy. At short exposure time, the image sensor saturation as well as image brightness due to light reflection from rail surface both disappears.

\section{Image Noise in a Digital Camera}

The images captured by an imaging system are often accompanied by noise, which is normal in any system such as electronic devices. Image noise in digital cameras is a random variation in the intensity of a pixel for a grayscale image or a random change in the value of a specific color channel for a color image and is usually an aspect of electronic noise.

There are three common types of noise in an image captured by a digital camera: 1-random noise, 2-"Fixed pattern" noise, and 3-banding noise. Figure 6 shows the effects of three types of noise on an image captured from a normal smooth gray background.

Random noise is recognized according to a change in the light intensity or color of a pixel higher or lower than the actual light intensity. The pattern of random noise changes even if the camera setting is fixed.

Fixed pattern noise shows "hot pixels" in the image; hot pixels are defined as pixels whose intensity is much higher than the amplitude of the random noise. This noise has a unique pattern in the same camera setting.

Banding noise is a camera-dependent noise, and it appears when the camera reads digital sensor data. It depends on the camera model.

The characteristics of these types of noise are listed in Table 1. As addressed in the table, the random noise usually occurs at high ISO speed and short exposure time, banding noise happens in a susceptible camera, and the fixed pattern noise occurs at low ISO speed and long exposure time.

Banding and fixed pattern noises are among the controllable noises, and due to their fixed pattern, they can be easily eliminated, or it is possible to provide conditions at which they are less likely to occur. This operation can be done by identifying the camera's internal electronics and detecting noise pattern. These noises are less common in modern digital cameras and cause fewer image problems than random noise. Although random noise has a less amplitude than other noises, it is more difficult to eliminate the random noise and cannot be achieved without reducing image quality. Therefore, we consider random noise and its effects on the image here. 


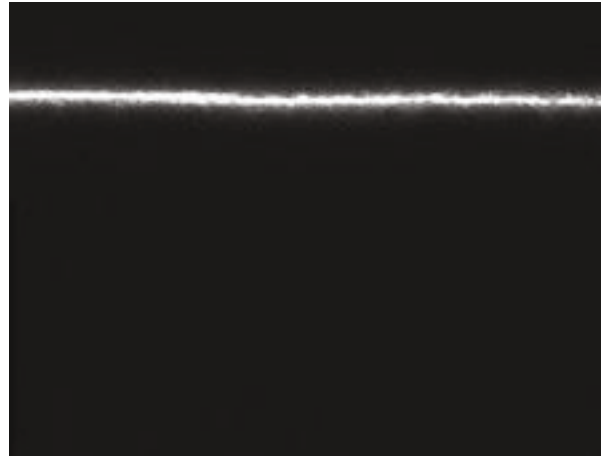

(a)

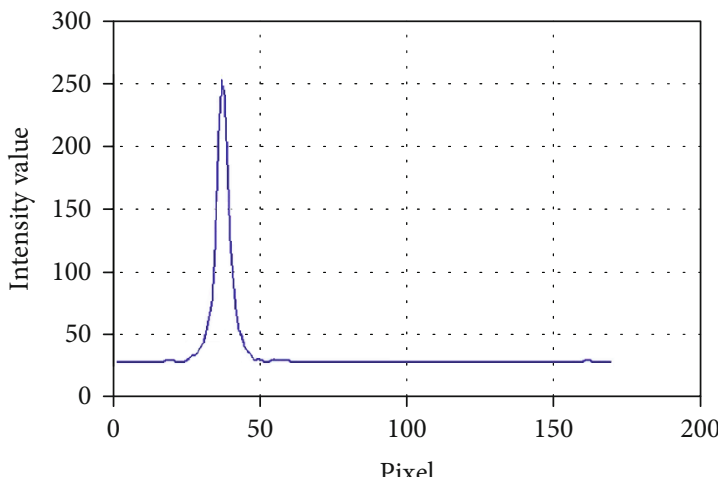

(b)

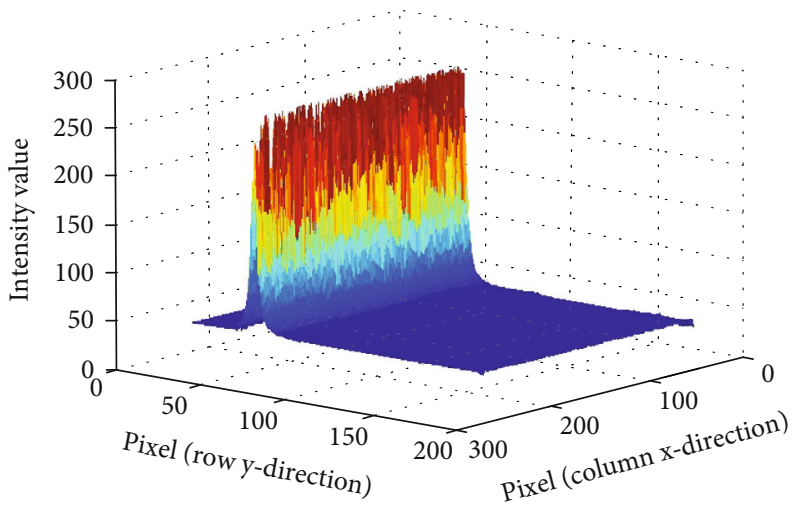

(c)

Figure 5: Laser stripe. (a) The captured image. (b) Intensity of the laser in a specified column of the captured image (in grayscale mode). (c) Image intensity.

\section{Laser Peak Detection Using Continuous Wavelet Transformation}

A wavelet is defined as a function $\psi(t)$ in $L^{2}(R)$ such as $\psi(t)$ satisfies the following condition:

$$
\int_{-\infty}^{+\infty} \psi(t) d t=0
$$

Continuous wavelet transform for function $f(t)$ is defined at point $\left(t_{0}, s\right)$ as follows:

$$
W[f, \psi]\left(t_{0}, s\right)=\left\langle f, \psi_{t_{0}, s}\right\rangle=\frac{1}{\sqrt{s}} \int_{-\infty}^{+\infty} f(t) \psi\left(\frac{t-t_{0}}{s}\right) d t
$$

where $\psi_{t_{0}, s}(t)$ is $\psi(t)$ which is shifted as $t_{0}$ and scaled tos.

$$
\psi_{t_{0}, s}(t)=\frac{1}{\sqrt{s}} \psi\left(\frac{t-t_{0}}{s}\right)
$$

Results of the continuous wavelet transform coefficients show the pattern of peaks distribution. By utilizing such pattern, the location and power of the peaks in the input signal $f(t)$ can be robustly obtained. This is done based on the similarity of a segment in the input signal including the peak and a scale of wavelet $\psi_{t_{0}, s}$ which is obtained through Gaussian pattern of the laser intensity. By varying $s$ in $\psi_{t_{0}, s}$, wavelets with different widths are provided. Here, depending on the nature of the laser pattern, the wavelet width is determined.

5.1. Appreciate Wavelet Selection. The most similar pattern to a laser reflected pattern from the rail surface can be obtained through a Gaussian pattern. The Gaussian function has several orders, which each of them have specific shape. Here, the Ricker wavelet known as Mexican hat wavelet that is the negative normalized second derivative of a Gaussian function is utilized for the laser peak detection (shown in Figure 7).

$$
\psi(t)=\frac{2}{\sqrt{3 \sigma} \pi^{1 / 4}}\left(1-\left(\frac{t}{\sigma}\right)^{2}\right) e^{-\frac{t^{2}}{2 \sigma^{2}}}
$$

where $\sigma$ is the standard deviation of the Gaussian function.

5.2. Range of Scales. One of the most important factors in using wavelet transform to detect the peak of the signal is to determine the range of scales required for the transform. Range of scales is obtained by the width of Gaussian pattern due to laser intensity in the image taken by the laser 


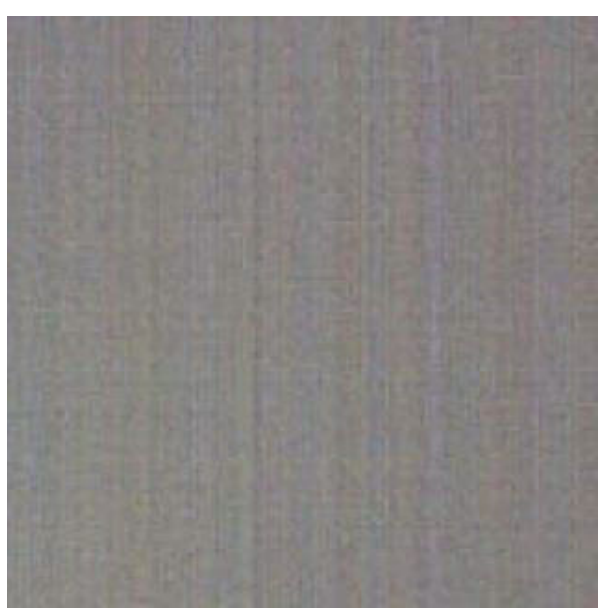

(a)

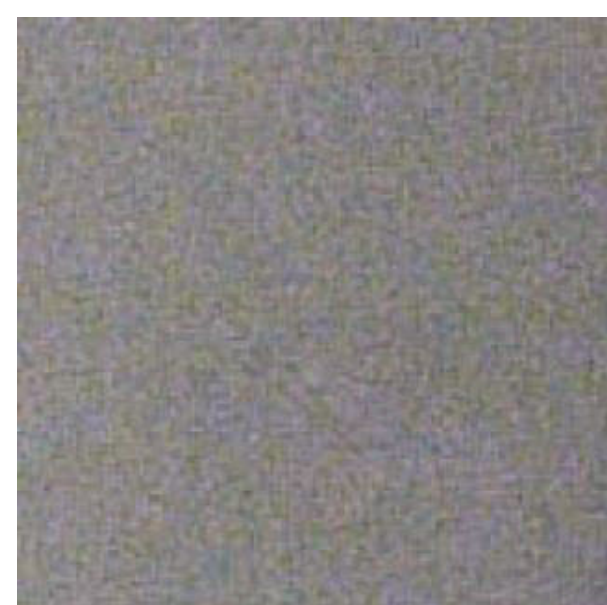

(b)

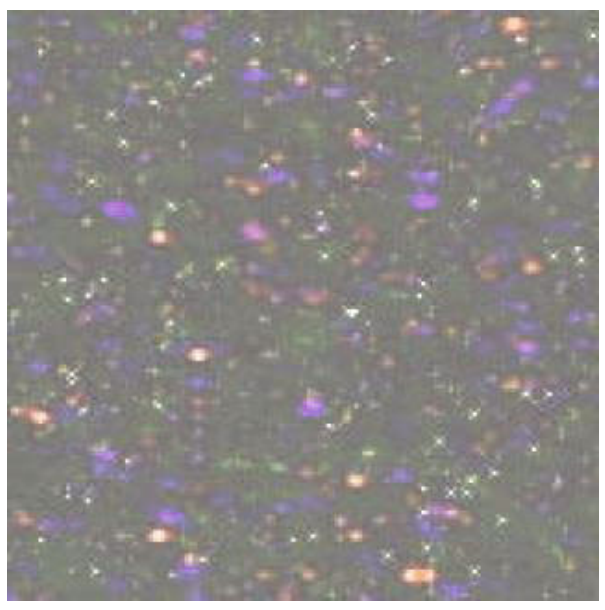

(c)

FIgURE 6: Common noise types in an image captured by a digital camera. (a) Banding noise. (b) Random noise. (c) "Fixed pattern" noise.

measurement system, so that all possible widths are covered. Each triangulation-based laser measurement system has a specific working range (since lens focus and field of view are limited). The working range is defined as the distance between the laser measurement system and the object to be measured. The minimum and maximum of this distance are supposed to be $r_{\min }$ and $r_{\max }$, respectively. The camera pin-hole model (shown in Figure 8) is defined by

$$
\left[\begin{array}{c}
x_{c} \\
y_{c} \\
z_{c} \\
1
\end{array}\right]=\left[\begin{array}{cc}
R & t \\
O_{3}^{T} & 1
\end{array}\right]\left[\begin{array}{c}
x_{w} \\
y_{w} \\
z_{w} \\
1
\end{array}\right]
$$

where $\left(x_{w}, y_{w}, z_{w}\right)$ is assumed to be the coordinates of a point $P$ on the object in the world coordinate system (WCS), and its corresponding coordinates are represented by $\left(x_{c}, y_{c}, z_{c}\right)$ in the camera coordinate system (CCS). $R$ and $t$ are the rotation matrix and translation vector, respectively.
TABLE 1: Properties of three types of noises in a digital camera.

\begin{tabular}{lccc}
\hline Noise type & Random & Banding & Fixed pattern \\
\hline Exposure & Short & Camera-dependent & Long \\
ISO speed & High & Camera-dependent & Low \\
\hline
\end{tabular}

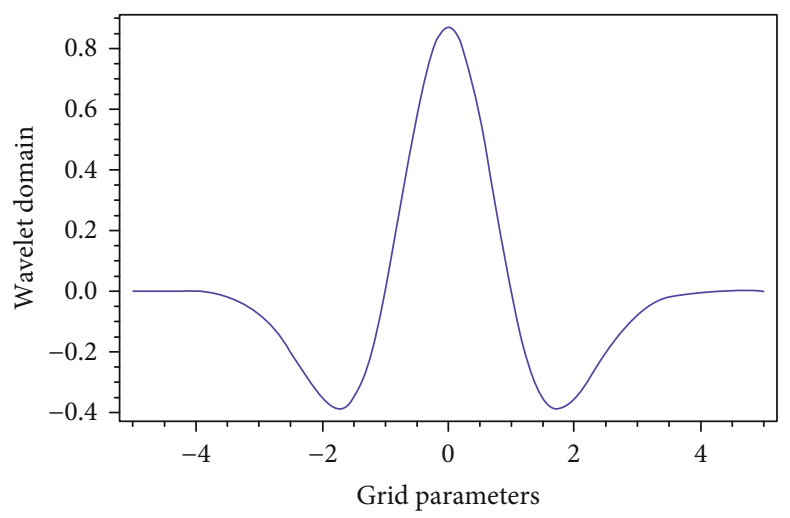

FIgURE 7: Distribution of the laser light reflected from a rail surface. 


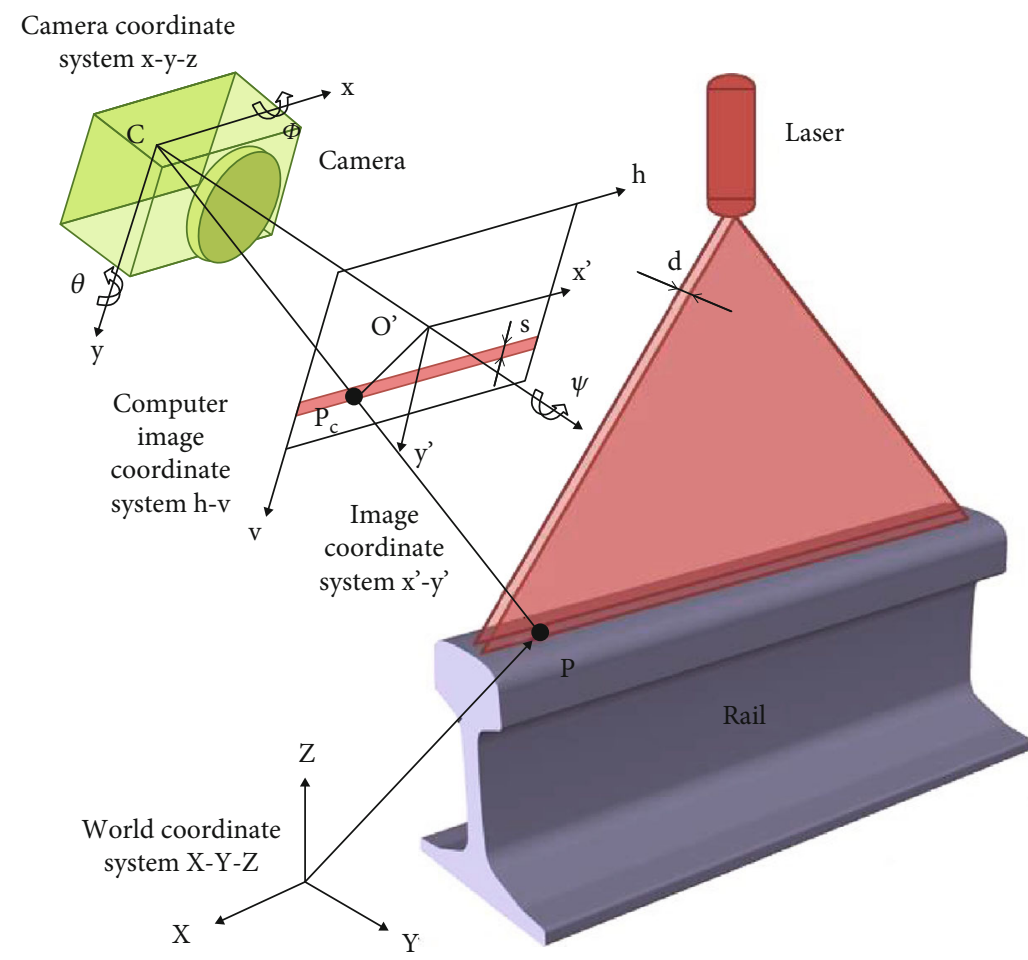

FIGURE 8: Laser and camera model for a rail corrugation measurement system (based on triangulation principle).

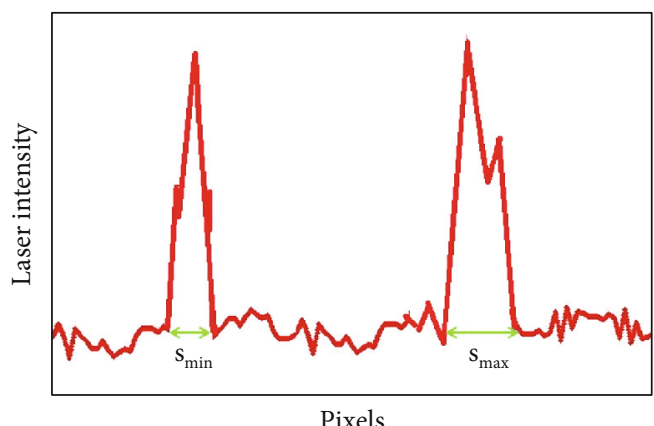

(a)

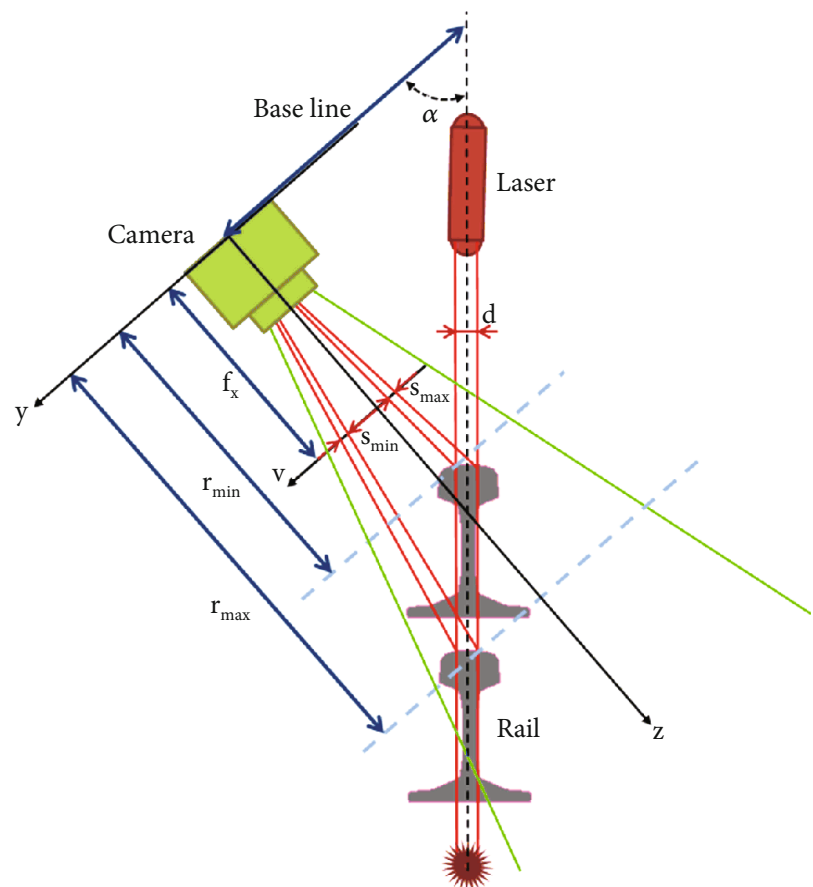

(b)

FIGURE 9: Relation between the laser diameter $d$ and scale of the wavelet $s$. (a) Minimum and maximum of the scales, $s_{\min }$ and $s_{\text {max }}$. (b) Geometrical configuration of the rail, camera, and the scale $s$. 
TABLE 2: Existing methods for laser peak detection in subpixel accuracy [10-13].

\begin{tabular}{lc}
\hline Estimation method & Formulae \\
\hline $\begin{array}{l}\text { Gaussian approximation } \\
\text { Center of mass }\end{array}$ & $\widehat{\delta}=1 / 2 \cdot(\ln (a)-\ln (c) / \ln (a)+\ln (c)-2 \cdot \ln (b))$ \\
$\widehat{\delta}=c-a / a+b+c$ & $\widehat{\delta}=\left\{\begin{array}{cc}g(i) / g(i)-g(i+1), & f(i+1)>f(i-1) \\
g(i-1) / g(i-1)-g(i), & f(i+1)<f(i-1) \\
\widehat{B}=1 / 2 . a-b / c-2 . b+a\end{array}\right.$ \\
Parabolic estimator & $\widehat{X}= \begin{cases}x-a-c / 2(b-a), & c<a \\
x-a-c / 2(b-c), & \text { otherwise } \\
\text { Linear interpolation } & \widehat{X}=x_{0}-y_{0} \cdot\left(x_{1}-x_{0}\right) / y_{1}-y_{0}\end{cases}$ \\
\hline
\end{tabular}

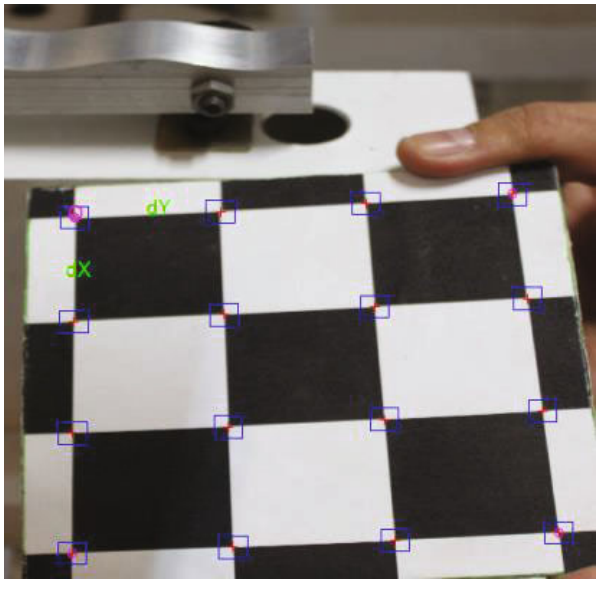

(a)

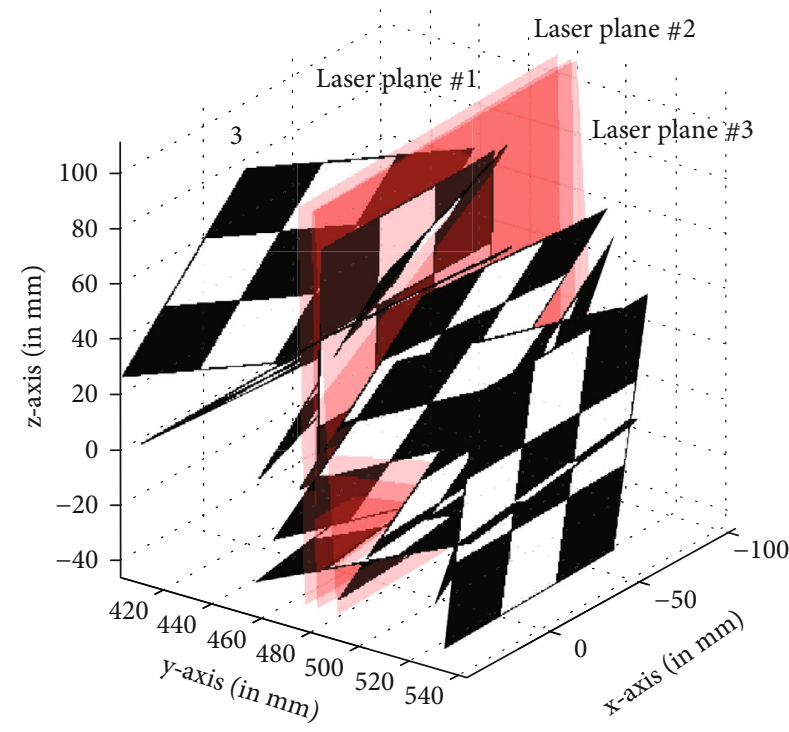

(b)

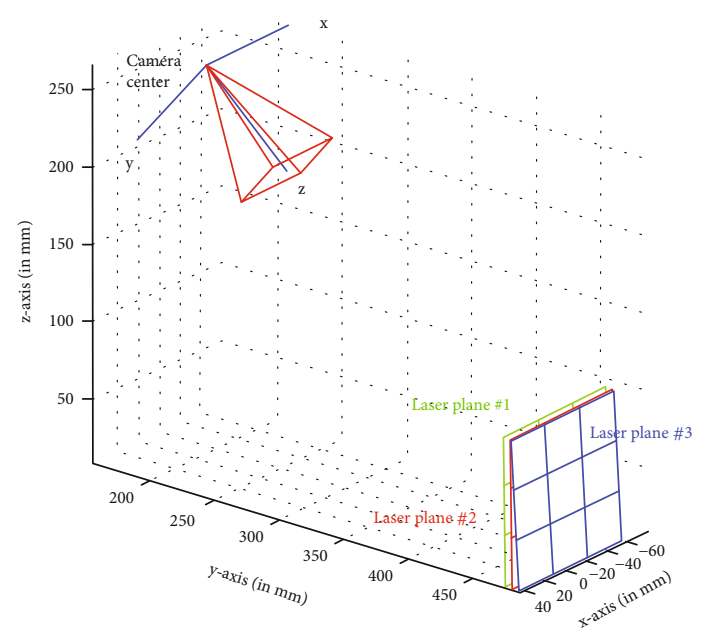

(c)

FIgure 10: Calibration of the CMS. (a) The calibration checkerboard plane. (b) Different positions of the calibration plane. (c) The laser planes extracted thorough surface fitting. 


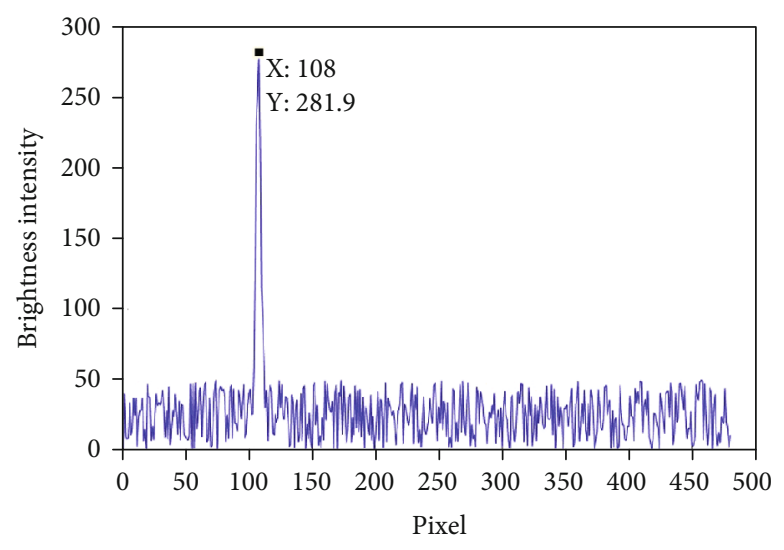

(a)

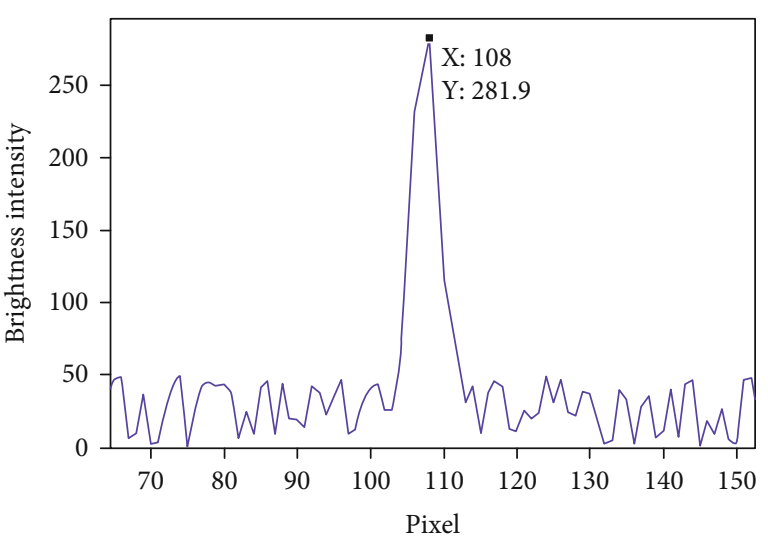

(b)

FIGURE 11: Synthetic signal generated by MATLAB software to simulate the laser light intensity in a row/column of a captured image. (a) The raw signal. (b) Close view of the signal peak (region of interest).

Then, we have

$$
\left[\begin{array}{l}
h \\
v \\
1
\end{array}\right]=\left[\begin{array}{ccc}
f_{x} & \alpha_{c} & h_{0} \\
0 & f_{y} & v_{0} \\
0 & 0 & 1
\end{array}\right]\left[\begin{array}{c}
x_{c} / z_{c} \\
y_{c} / z_{c} \\
1
\end{array}\right],
$$

where $f_{x}$ and $f_{y}$ are defined as focal lengths in directions $x$ and $y$, respectively. $h_{o}$ and $v_{o}$ are coordinates of the center of the image coordinate system. $\alpha_{c}$ shows the skew between $h$ and $v$ axes. After applying mathematical operations,

$$
z_{c} \cdot\left[\begin{array}{c}
h \\
v \\
1
\end{array}\right]=\left[\begin{array}{ccc}
f_{x} & \alpha_{c} & h_{0} \\
0 & f_{y} & v_{0} \\
0 & 0 & 1
\end{array}\right]\left[\begin{array}{cc}
R_{1} & t_{1} \\
O_{3}^{T} & 1
\end{array}\right]\left[\begin{array}{c}
x_{w} \\
y_{w} \\
z_{w}
\end{array}\right] .
$$

The above parameters are known as internal and external parameters of a camera and can be determined by the existing methods [34-36]. Also, the nonlinearity of the camera model can be taken as lens distortion in the above model, but without loss of generality, the lens distortion is not taken into account; as in new lenses, the distortion is negligible and has small effect on the results. Also, the range of scales is an approximate parameter and defined by a margin of safety.

According to Figure 8, the diameter of the laser emitter is denoted by $d$ (its unit is $\mathrm{mm}$ ). The laser light that emits from laser diode source is modeled as a flat plane as follows:

$$
\left(x_{w}-x_{l}\right) \cdot n_{x}+\left(\mathrm{y}_{w}-y_{l}\right) \cdot n_{y}+\left(\mathrm{z}_{w}-z_{l}\right) \cdot n_{z}=0
$$

$x_{l}, y_{l}$, and $z_{l}$ are the coordinates of a point on the laser plane, and $n_{x}, n_{y}$, and $n_{z}$ are the components of the normal vector of the laser plane. Some techniques to extract the laser plane parameters are explained in [37-41].

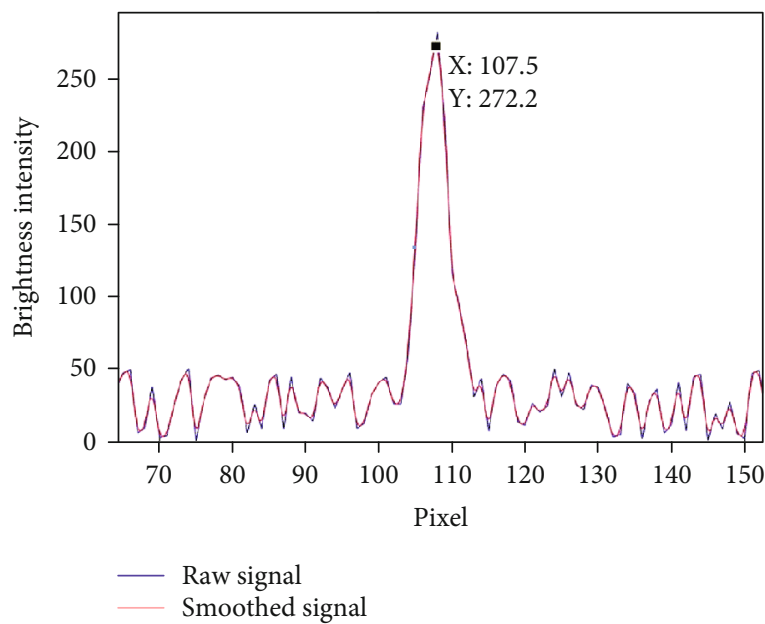

FIGURE 12: Smoothed signal after filtering and peak detected.

When the camera and laser parameters were determined, the range of scales can be obtained to be.

$$
R=\left[\begin{array}{lll}
1 & 0 & 0 \\
0 & 1 & 0 \\
0 & 0 & 1
\end{array}\right], t=\left[\begin{array}{l}
0 \\
0 \\
0
\end{array}\right] .
$$

As can be seen in Figure 9, the minimum and maximum of the scales are defined as follows:

$$
\begin{aligned}
& s_{\text {min }}=\frac{f_{x}}{r_{\text {max }}} \times d \times \sin (\alpha), \\
& s_{\text {max }}=\frac{f_{x}}{r_{\text {min }}} \times d \times \sin (\alpha),
\end{aligned}
$$

where $\alpha=\arctan \left(n_{y} / n_{z}\right)$ denotes the angle between camera and laser plane at origin. It can be determined from the normal vector of laser plane. $f_{x}$ is the focal length in the $x$-direction, if a row selected. So, the range of scales is 


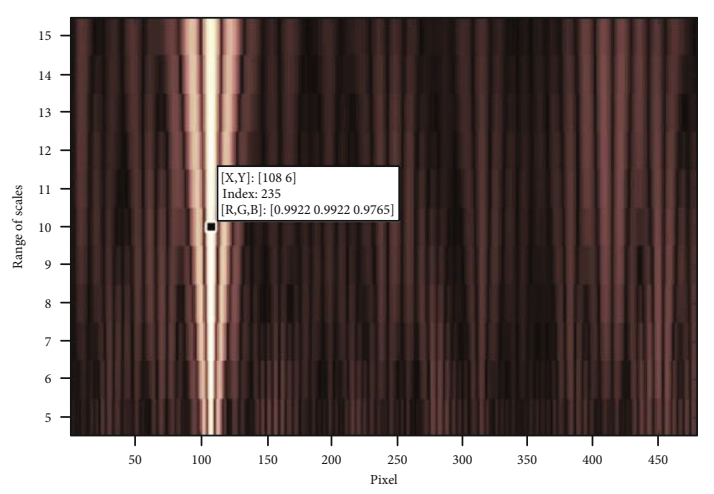

(a)

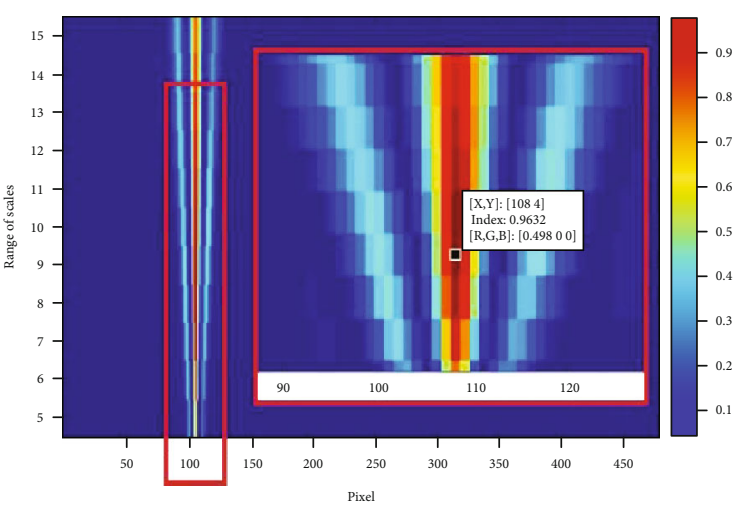

(b)

FIGURE 13: Signal peak detection in pixel accuracy using wavelet transform. (a) Wavelet transform coefficients and the range of scales. (b) Scalogram of wavelet transform.

defined between the minimum of scale denoted as $s_{\min }$ and the maximum of scale denoted as $s_{\max }$. In practice, due to different optical surface properties, a margin of safety about $25 \%$ of the range is considered in upper and lower border.

5.3. Achieving Subpixel Accuracy. All the methods summarized in Table 2 are suitable for the laser peak detection at subpixel accuracy, but for implementing the wavelet transform capability for laser peak detection at subpixel accuracy, the wavelet coefficients of appreciate wavelet in the range of scales should be taken into calculation. As the energy distribution of a laser stripe follows a Gaussian pattern, a waveletbased template matching can robustly detect the laser peak in the image. Suppose $W[f, \psi]\left(t_{0}, s\right)$ as the wavelet transform coefficients of $f(\mathrm{t})$ in the $s$-scale, in this method, there is no need to smooth or bias the input signal before utilization. To achieve the subpixel accuracy, the wavelet coefficients which are greater than a threshold are selected:

$$
W_{\text {Sel }}[f, \psi]\left(t_{0}, s\right)=W[f, \psi]\left(t_{0}, s\right)>T_{\text {threshold }} .
$$

Threshold $T$ can be obtained through the energy of laser Gaussian pattern. Now, by use of the selected wavelet coefficients, laser peak will be detected in subpixel accuracy as follows:

$$
t_{\text {peak }}=\frac{\sum_{i=1}^{n} t_{0_{i}} \cdot W_{\text {Sel }_{i}}[f, \psi]\left(t_{0_{i}}, s_{i}\right)}{\sum_{i=1}^{n} W_{\text {Sel }_{i}}[f, \psi]\left(t_{0_{i}}, s_{i}\right)},
$$

where $t_{0 i}$ is the shift of $i^{\prime}$ th wavelet coefficient $W_{\text {Sel }_{i}}[f, \psi]$ $\left(t_{0_{i}}, s_{i}\right)$ in scale of $s_{i}$. Accordingly, the laser peak detection in subpixel accuracy is accomplished. In fact, the proposed method is a variation of center of the mass method.

\section{Experimental Results}

Methodology of calibration and error analysis is described for the fabricated set up in this section. Subsequently, experimental results are presented and discussed.
TABLE 3: Result of 100 tests on synthetic data (100 different random noisy signals generated by MATLAB software).

\begin{tabular}{lcc}
\hline Peak detection & $\begin{array}{c}\text { Accuracy } \\
\text { (pixel) }\end{array}$ & $\begin{array}{c}\text { Processing time } \\
(\mathrm{sec})\end{array}$ \\
\hline Center of gravity method & \pm 0.0154 & $3.6612 e-5$ \\
Center of gravity method after & \pm 0.0121 & 0.0031 \\
filtering & \pm 0.0062 & 0.1823 \\
Proposed method & & \\
\hline
\end{tabular}

6.1. System Calibration. The presented CMS is modeled as a laser camera triangulation system. In this system, each of the components including camera and laser is separately modeled, but, just a single checker board plane is utilized for calibration.

Camera is considered as a pinhole model. The camera parameters (rotation matrix, translation vector, lens deflection, axis skew and ...) are extracted from the corresponding point pairs by minimizing the summation of the squared errors. Corresponding pairs are defined as pairs between points on the calibration plane and their corresponding points on the image plane (shown in Figure 10(a)). Also, the effect of nonlinear deformation due to the lens distortion is considered. By utilizing an appropriate software (such as MATLAB Camera Calibration Toolbox [42]), the checkerboard pattern can be automatically detected.

A laser can be considered as a planner plane; so, the model of the laser follows equation of a plane. In order to obtain the parameters of the three laser planes, the checker board plane is placed at an arbitrary position in front of the camera. While the laser emitters are on, camera uses a snapshot from them (as shown in Figure 10(b)). Therefore, the patterns of checkerboard and laser planes are captured together. 3D coordinates of the laser points are then obtained through reprojection of the laser peaks detected on the image to the calibration board. Laser rays passing from the center of the camera coordinate system and laser detected peaks on the image plane are intersected by the checkerboard plane. Then, the parameters of the lasers' planes are obtained thorough surface fitting (shown in Figure 10(c)). By utilizing 


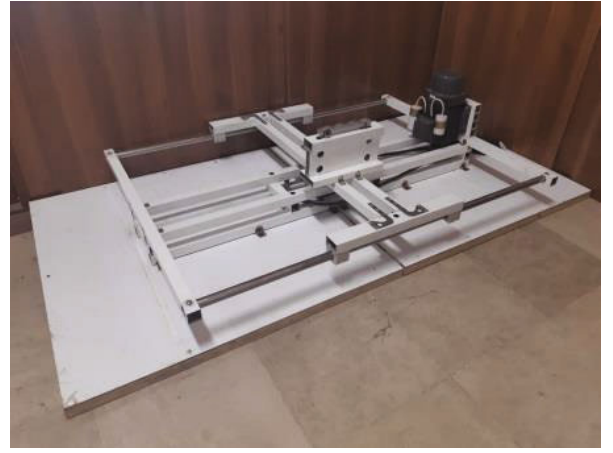

(a)

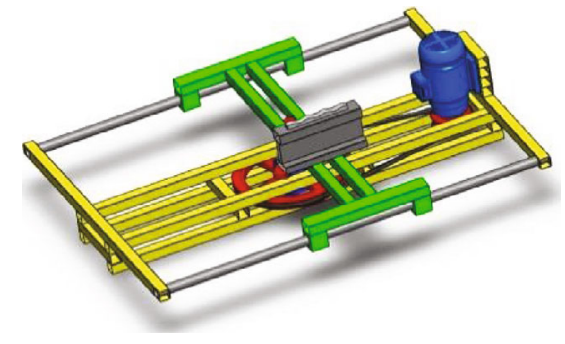

(b)

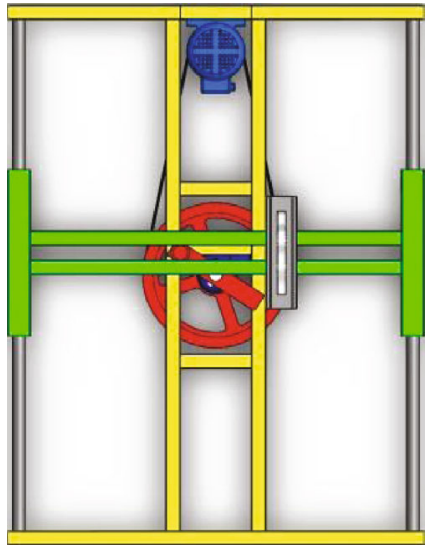

(c)

Figure 14: Rail simulator. (a) Manufactured rail simulator. (b, c) CAD model of rail simulator.

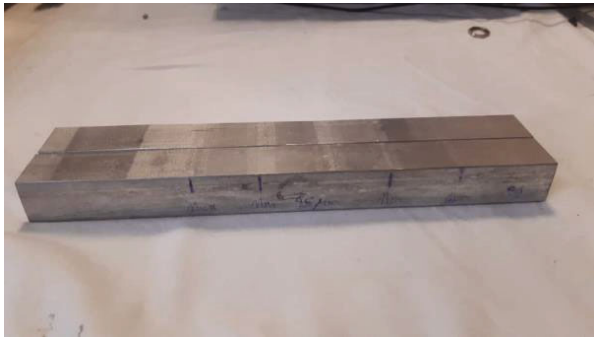

(a)

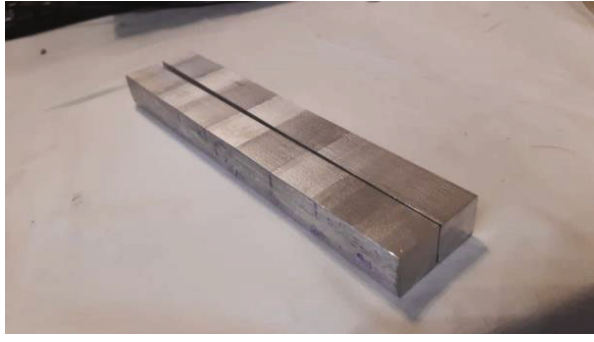

(b)

Figure 15: Samples for simulating the rail corrugation. (a, b) Two views of the manufactured samples for simulating the rail corrugation with wavelength of $5 \mathrm{~cm}$ and amplitudes of 50 and 100 micrometers.

the above method, the laser camera system is calibrated using only a single plane, and no extra equipment is required.

6.2. Algorithm Evaluation. In order to evaluate accuracy and performance of the proposed method, some experiments were performed on both synthetic and real data. At first, a synthetic discrete signal was created. This signal is a random noisy signal generated using random function of the MATLAB software. The signal length is selected to be 480 pixels, which is equal to the number of rows in an image with $640 \times 480$ resolution (480p $4: 3$ aspect ratio). In order to simulate the laser peak in the corresponding signal, a Gaussian pattern was generated using the related function in MATLAB software. The width of the pattern (the stan- dard deviation of Gaussian kernel) was selected such that to be near to the laser width in the image captured by an ordinary laser measurement system. The amplitude of the peak was selected so that the signal-to-noise ratio is similar to the reality. In fact, the values of the signal represent the light intensity of each pixel in a specific color channel or in a grayscale image. This high-frequency noisy signal integrated by the Gaussian pattern is shown in Figure 11(a).

The signal has been defined such that the peak was located at 107.5 pixels of the signal. But as can be seen in Figure 11(b), a close view of the signal shows that if no preprocessing is performed, the peak is detected at pixel 108 . This phenomenon indicates that, in the presence of noise, a large error in peak detection may occur. In a triangulation- 


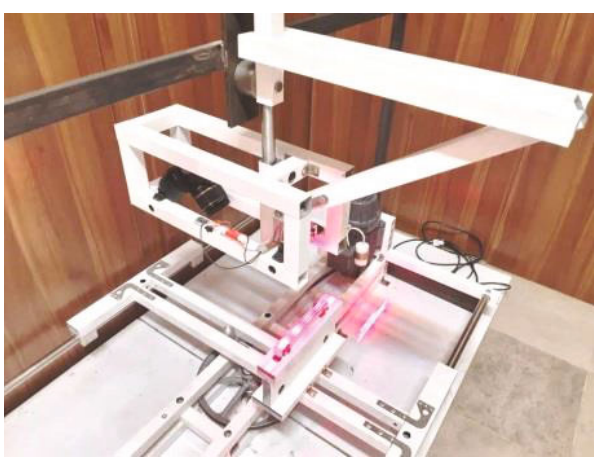

(a)

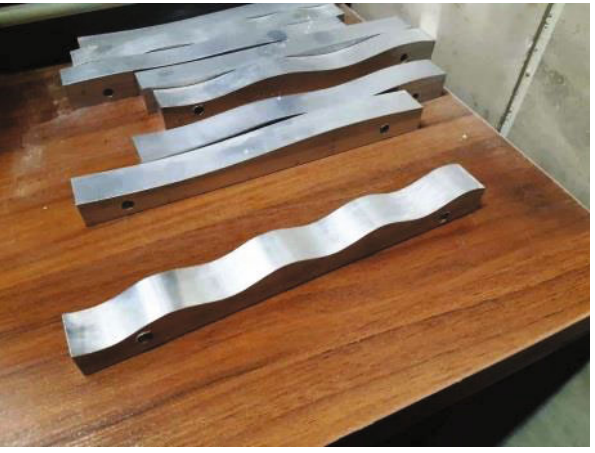

(b)

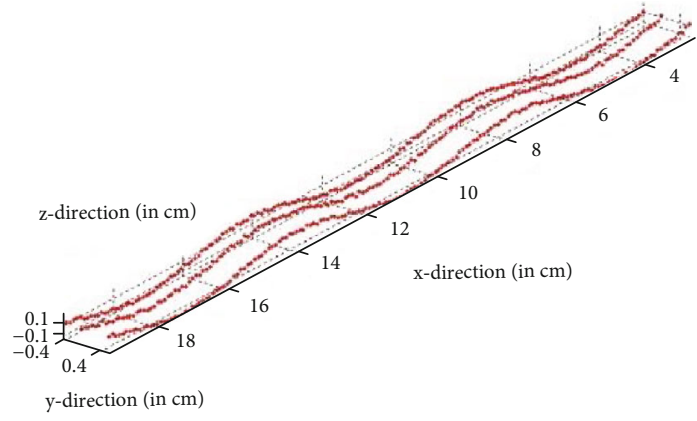

(c)

FIGURE 16: Designed and manufactured prototype based on the laser triangulation method for the rail corrugation measurement [97]. (a) Prototype and its test rig for simulating the corrugated rail as a moving object. (b) Samples produced by CNC machine for simulating the rail corrugation. (c) $3 \mathrm{D}$ laser reconstruction of the sample.

based measurement system, detecting the laser stripe peak in pixel accuracy causes large errors in measurement; hence, all of the available methods try to detect laser peak in subpixel accuracy.

To detect laser peak by use of available methods, its better, the signal to be smoothed using a proper filter. Hence, a lowpass filter is designed; here, the filter coefficients are determined through Fourier transform and based on the nature of the signal noise. After designing the filter, the corresponding signal is smoothed by use of the designed filter and the laser peak detected through the center of gravity method in subpixel accuracy. Figure 12 shows the smoothed signal and the detected peak. The difference between the detected signal peak and its real value is 0.012 in subpixel accuracy.

Now, the signal peak is detected by use of the proposed method. Here, the range of scales determined through the width of generated Gaussian pattern which is integrated in the synthetic signal (Gaussian pattern width is equal to 10 units, scales range $s_{\min }$ and $s_{\max }$ defined as 5 and 15 pixels, respectively). As shown in Figure 13(a), the laser peak is detected at 108, robustly. Scalogram of the signal is illustrated Figure 13(b). To detect the signal peak in subpixel accuracy, first, the wavelet transform coefficients are calculated. Threshold for selecting the best coefficients can be determined through the calculation of the area under an ordinary laser Gaussian pattern in the synthetic data. Then, the laser peak will be detected through Eq. (13). The difference between the detected signal peak and its real value is 0.006 in subpixel accuracy. The above test was performed on 100 signals with signalto-noise ratio of about $30 \mathrm{~dB}$. The experimental results
TABLE 4: Specification of the proposed corrugation measurement prototype and the range of scales.

\begin{tabular}{lc}
\hline Parameters & Value \\
\hline Camera resolution & $4752 \times 3168$ pixels \\
Camera type & Canon $500 \mathrm{D}$ \\
Camera focal length @ $24 \mathrm{~mm}$ & 5106 and 5107 pixels \\
& $x$ and $y$ direction \\
Laser wave length & $685 \mathrm{~nm}$ \\
Laser diameter & $2 \mathrm{~mm}$ \\
Angle between laser and camera, $\alpha$ & 42 degrees \\
Working range, $r_{\min }$ & $35 \mathrm{~cm}$ \\
Working range, $r_{\max }$ & $55 \mathrm{~cm}$ \\
Range of scales, $s_{\min }$ & 10.5 pixels \\
Range of scales, $s_{\max }$ & 21.5 pixels \\
\hline
\end{tabular}

TABLE 5: Result of 40 tests on 4 different samples manufactured by $\mathrm{CNC}$ machine to simulate the corrugation of the rail (with wavelength of 5 and $10 \mathrm{~cm}$ and amplitude of 50 and 100 micrometer).

\begin{tabular}{lcc}
\hline \multirow{2}{*}{ Corrugation measurement } & \multicolumn{2}{c}{ Error $(\mathrm{mm})$} \\
& Wavelength & Amplitude \\
\hline Center of gravity method & \pm 0.19 & \pm 0.009 \\
Center of gravity method after filtering & \pm 0.17 & \pm 0.008 \\
Proposed method & \pm 0.11 & \pm 0.005 \\
\hline
\end{tabular}




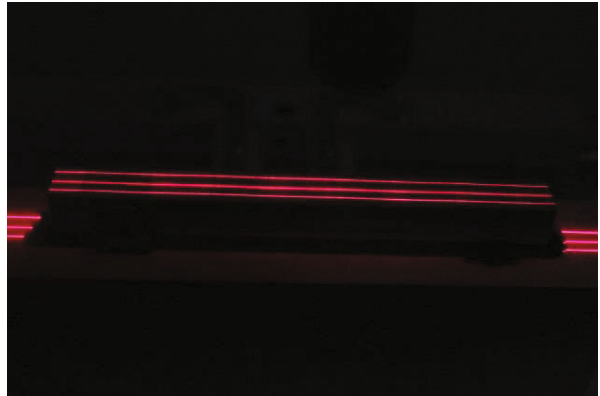

(a)

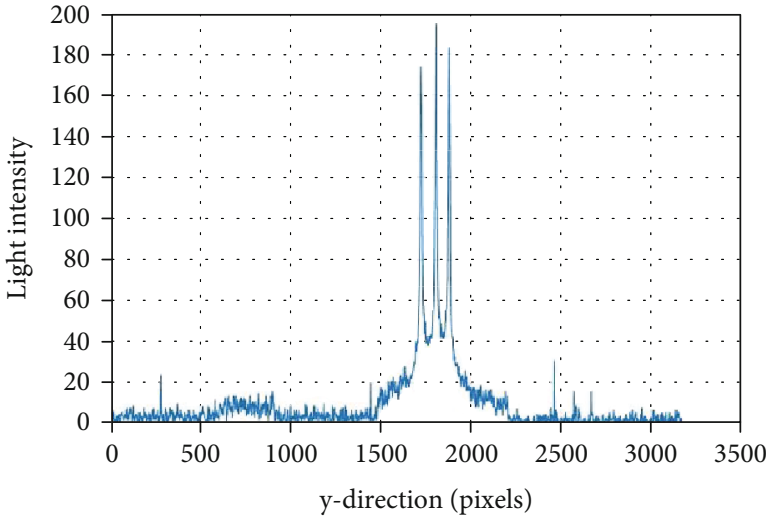

(b)

FIGURE 17: Image captured from a sample of corrugated rail (wavelength of $5 \mathrm{~cm}$ and amplitude of 50 micrometer). (a) Captured image from 3 laser beams. (b) Light intensity of each laser and its power $(S / N \approx 30 \mathrm{~dB})$.

including the accuracy and computation time are listed in Table 3. As can be seen by using the proposed method, the laser peak was detected in subpixel accuracy more robust than the filtering method.

It should also be added that the accuracy and speed of detection also depend on the system settings including the camera and the digital processing system. As shown in Table 3, a comparison between the proposed laser peak detection and two other popular peak detection methods performed by a similar digital processing system shows that the accuracy can be doubled with almost the same processing time. This is a remarkable benefit for the field measurements.

To simulate a real field test, it was decided to design and manufacture a rail simulator that could simulate conditions similar to the actual conditions that occur when the proposed corrugation measurement system measures a rail corrugation. For this purpose, the rail is oscillating, and the measuring system is fixed. Figure 14 presents the proposed rail simulator.

The proposed rail simulator is based on the Scotch yoke mechanism and operates in a reciprocating manner that moves the rail for a maximum speed of up to $30 \mathrm{~km} / \mathrm{h}$.

Also, to determine the characteristic of rail corrugation, some artificial samples have been made by a CNC machine as like as a sine wave to simulate a corrugated rail (the accuracy of the production is about \pm 20 micrometers). Then, the sine wave amplitude is measured again using a micrometer to confirm the accuracy of production. The samples for simulating the rail corrugation have been produced by wavelength of 5 and $10 \mathrm{~cm}$ and amplitude of 50 and 100 micrometer. Figure 15 shows the produced samples with wavelength of $5 \mathrm{~cm}$ and amplitudes of 50 and 100 micrometers.

An experiment was also designed to get real data. As shown in Figure 16(a), a prototype based on the laser triangulation, for the rail corrugation measurement, was designed and manufactured. The specifications of the corrugation measurement prototype and its corresponding range of scales are depicted in Table 4. Camera parameters are computed by use of Camera Calibration Toolbox for MATLAB [43]. Range of scales was determined through Eqs. (9) and (10) by considering the focal length of the camera, working range, and the angle between camera and laser source of the laser measurement system.

As shown in Figure 16(b), some samples for the simulating rail corrugation were produced by $\mathrm{CNC}$ (computer numerical control) machine. Each sample has unique wavelength and domain that indicates the specification of a rail corrugation type. By use of the laser measurement prototype developed for rail corrugation measurement, the wavelength and amplitude of the samples are measured. The 3D reconstructed points of the sample are depicted in Figure 16(c). By comparing the exact and measured wavelength and amplitude of the fabricated specimens, the error of the rail corrugation measurement can be calculated. Also, a method for evaluation and analysis of digitizing errors of a laser scanning system has been described in [44]. Table 5 indicates the results of measurements error for 40 tests on 4 different samples manufactured by CNC machine to simulate the corrugation of rail (with wavelength of 5 and $10 \mathrm{~cm}$ and amplitude of 50 and 100 micrometer).

In addition to the tests carried out on the corrugated rail samples with amplitudes of 50 and 100 micrometers, extra experiments were performed on the other CNC manufactured samples with the wavelength of 5,10 , and $15 \mathrm{~cm}$ and the amplitudes of 1,2 , and $3 \mathrm{~mm}$. Results show that the accuracy of measurement for theses amplitudes and wavelengths is in the same percentage order as that was measured before for the corrugated rail samples.

Figure 17 shows the image captured by the proposed rail corrugation measurement system (wavelength of $5 \mathrm{~cm}$ and amplitude of 50 micrometer). The rail corrugation should be measured on running surface of the rail. The use of three laser planes enables the CMS to cover the whole running surface of the rail. As can be seen, three laser planes are utilized for the laser peak detection and $3 \mathrm{~d}$ reconstruction, and the light intensity of each laser and its power $(\mathrm{S} / \mathrm{N} \approx 30 \mathrm{~dB})$ is clear. Figure 18 shows results of rail corrugation measurement for two samples. The samples wavelengths are $5 \mathrm{~cm}$ but their amplitudes vary between 50 and 100 micrometer. It is seen that results of the proposed method are reliable both in estimating the amplitude and the wavelength. 


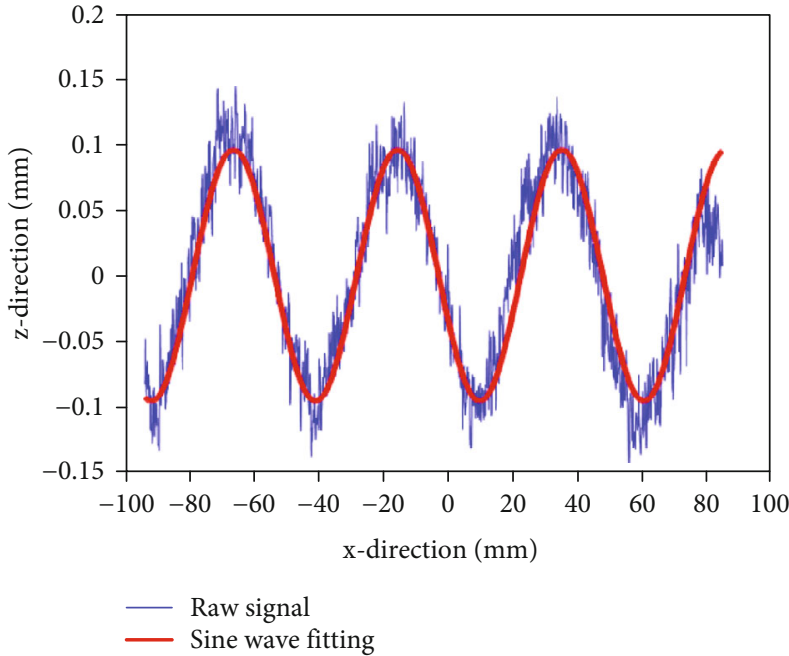

(a)

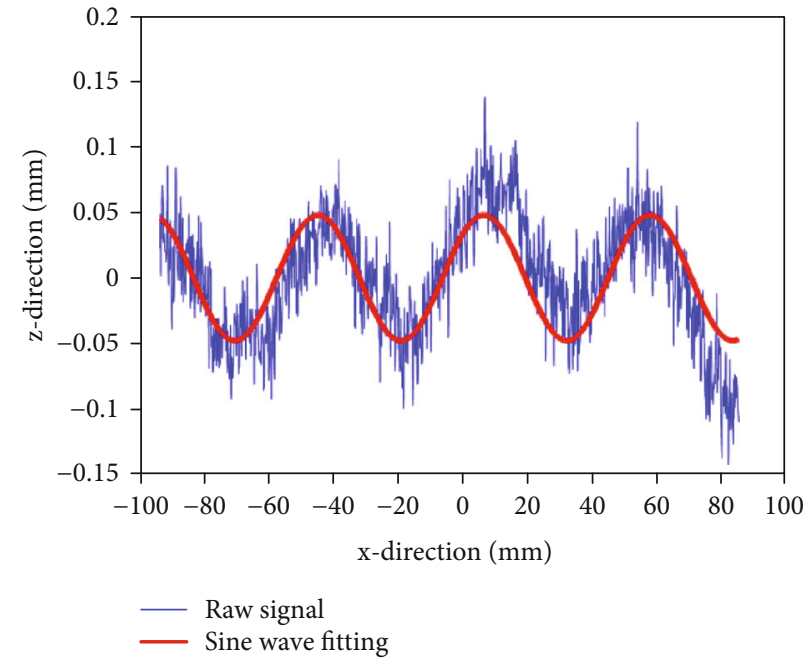

(b)

Figure 18: Result of rail corrugation measurement. (a) Wavelength of $5 \mathrm{~cm}$ and amplitude of 100 micrometer. (b) Wavelength of $5 \mathrm{~cm}$ and amplitude of 50 micrometer.

As addressed before, the corrugation is a quasisinusoidal irregularity, but as a mathematical model, it is considered as a sine wave. To extract the parameters of the corrugation, a sine wave fitting is required. For a simple sine wave fitting, two standard algorithms exist: 1-four-parameter sine wave fit (4PSF), known as IEEE-STD-1057, and 2-threeparameter sine wave fit (3PSF), known as IEEE-STD-1041. The main difference between the raised algorithms is in knowing the parameter $\lambda$ (the wavelength of the sine wave) which makes the problem linear or nonlinear for solving.

The proposed CMS has a moving reference, and the extracted 2-D profile data has an extra degree of freedom, i.e., rotation. In the presence of the rotation, the problem of sine wave fitting is completely nonlinear. The parameter $\lambda$ as corrugation wavelength is extracted at first through THE ellipse fitting method. Then, the rotation angle and other corrugation parameters are obtained by fitting a rotated sine wave to the 2-D profile data. Then, accurate parameters are extracted in an iterative manner [9].

\section{Conclusions}

Rail corrugation as an important source of noise and vibration in railways and subways should be continuously monitored, especially in public transportation. The existing measuement systems for the rail assessment use the laser triangulationbased corrugation measurement method. The accuracy of such systems is quietly depends to the algorithm utilized for the laser peak detection.

In this paper, we applied the Gaussian wavelet transform to detect the laser peaks, because the laser peak distribution follows a Gaussian pattern. The wavelet transform is capable of detecting the laser peak by pattern matching in a robust and rapid process. To achieve the subpixel accuracy, the wavelet transform coefficients were utilized.

The accuracy of the proposed method for synthetic data is 0.0062 pixels that in contrast with the filtering methods with accuracy of 0.0121 pixels gives two times better results. Also, the prototype system for the rail corrugation measurement based on the proposed method allows measurements at $5 \%$ of the range (up to 5 microns).

Applying the new methodology based on the wavelet transform provided a new rapid and accurate measurement platform for the rail longitudinal profile measurement. Simulation results as well as the experimental outcomes showed that the proposed method is quite independent from the rail quality and less affected by the random noise. Thin conclusion will candidate this new methodology for the industrial application.

\section{Data Availability}

Data are available upon request.

\section{Conflicts of Interest}

The authors declare that they have no conflicts of interest.

\section{References}

[1] F. Wu, J. Mao, Y.-F. Zhou, and L. Qing, “Three-line structured light measurement system and its application in ball diameter measurement," Optik, vol. 157, pp. 222-229, 2018.

[2] B.-R. Lee and H.-C. Nguyen, "Development of laser-vision system for three-dimensional circle detection and radius measurement," Optik, vol. 126, no. 24, pp. 5412-5419, 2015.

[3] X. Hao, A. Sha, Z. Sun, W. Li, and Y. Hu, "Laser-based measuring method for mean joint faulting value of concrete pavement," Optik, vol. 127, no. 1, pp. 274-278, 2016.

[4] S. L. Grassie, "Measurement of railhead longitudinal profiles: a comparison of different techniques," Wear, vol. 191, no. 1-2, pp. 245-251, 1996.

[5] S. L. Grassie, "Short wavelength rail corrugation: field trials and measuring technology," Wear, vol. 191, no. 1-2, pp. 149160, 1996. 
[6] R. Lewis and A. Richards, "A new method for the routine measurement of rail head corrugations," Rail International, vol. 17, no. 2, 1986.

[7] M. Deepak, "Evaluation of track condition by monitoring of masses," Rail International, vol. 17, no. 86, 1986.

[8] S. Grassie, "Corrugation on Australian National: cause, measurement and rectification," in Fourth International Heavy Haul Railway Conference 1989: Railways in Action, Australia, January 1989.

[9] S. M. M. Gazafrudi, D. Younesian, and M. Torabi, "A high accuracy and high speed imaging and measurement system for rail corrugation inspection," IEEE Transactions on Industrial Electronics, vol. 68, no. 9, pp. 8894-8903, 2021.

[10] Y. Zhang, W. Liu, X. Li, F. Yang, P. Gao, and Z. Jia, “Accuracy improvement in laser stripe extraction for large-scale triangulation scanning measurement system," Optical Engineering, vol. 54, no. 10, article 105108, 2015.

[11] P. Perona and J. Malik, "Scale-space and edge detection using anisotropic diffusion," IEEE Transactions on Pattern Analysis and Machine Intelligence, vol. 12, no. 7, pp. 629-639, 1990.

[12] J. Forest, J. Salvi, E. Cubruja, and C. Pous, "Laser stripe peak detector for 3D scanners. A FIR filter approach," in Proceedings of the 17th International Conference on Pattern Recognition, 2004. ICPR 2004, Cambridge, UK, August 2004.

[13] D. K. Naidu and R. B. Fisher, "A comparative analysis of algorithms for determining the peak position of a stripe to subpixel accuracy," in BMVC91, pp. 217-225, Springer, 1991.

[14] J. Forest Collado, New methods for triangulation-based shape acquisition using laser scanners, Universitat de Girona, 2004.

[15] A. Huertas and G. Medioni, "Detection of intensity changes with subpixel accuracy using Laplacian-Gaussian masks," IEEE Transactions on Pattern Analysis and Machine Intelligence, vol. PAMI-8, no. 5, pp. 651-664, 1986.

[16] M. Subbarao and Tao Choi, "Accurate recovery of threedimensional shape from image focus," IEEE Transactions on Pattern Analysis and Machine Intelligence, vol. 17, no. 3, pp. 266-274, 1995.

[17] Z. Lv and Z. Zhang, "Build 3D scanner system based on binocular stereo vision," Journal of Computers, vol. 7, no. 2, pp. 399404, 2012.

[18] Y. Li, J. Zhou, F. Huang, and L. Liu, "Sub-pixel extraction of laser stripe center using an improved gray-gravity method," Sensors, vol. 17, no. 4, p. 814, 2017.

[19] M. A. G. Izquierdo, M. T. Sanchez, A. Ibañez, and L. G. Ullate, "Sub-pixel measurement of 3D surfaces by laser scanning," Sensors and Actuators A: Physical, vol. 76, no. 1-3, pp. 1-8, 1999.

[20] C. Steger, "An unbiased detector of curvilinear structures," IEEE Transactions on Pattern Analysis and Machine Intelligence, vol. 20, no. 2, pp. 113-125, 1998.

[21] Z. Wei, F. Zhou, and G. Zhang, "3D coordinates measurement based on structured light sensor," Sensors and Actuators A: Physical, vol. 120, no. 2, pp. 527-535, 2005.

[22] L. Qi, Y. Zhang, X. Zhang, S. Wang, and F. Xie, "Statistical behavior analysis and precision optimization for the laser stripe center detector based on Steger's algorithm," Optics Express, vol. 21, no. 11, pp. 13442-13449, 2013.

[23] G. Xu, L. Sun, X. Li, J. Su, Z. Hao, and X. Lu, “Adaptable center detection of a laser line with a normalization approach using Hessian-matrix eigenvalues," Journal of the Optical Society of Korea, vol. 18, no. 4, pp. 317-329, 2014.
[24] H.-Y. Cai, Z.-D. Feng, and Z.-H. Huang, "Centerline extraction of structured light stripe based on principal component analysis," Chinese Journal of Lasers, vol. 42, no. 3, article 0308006, 2015.

[25] Q. Sun, J. Chen, and C. Li, "A robust method to extract a laser stripe centre based on grey level moment," Optics and Lasers in Engineering, vol. 67, pp. 122-127, 2015.

[26] L. Fengjiao, L. Xiaoqing, and L. Zhen, "A multi-scale analysis based method for extracting coordinates of laser light stripe centers," Acta Optica Sinica, vol. 11, pp. 111-116, 2014.

[27] H. Lei, D. Li, J. Wang, and B. Hu, "A method for fast detecting the center of structured light stripe," Journal of Huazhong University of Science and Technology, vol. 31, pp. 74-76, 2003.

[28] J. J. L. D. L. Heping, "New method for obtaining the center of structured light stripe," Computer Engineering and Applications, vol. 4, 2006.

[29] L. Z. L. S. F. Chang, "Laser stripe center extraction based on cross-correlation algorithm," Chinese Journal of Lasers, vol. 5, 2013.

[30] P. Du, W. A. Kibbe, and S. M. Lin, "Improved peak detection in mass spectrum by incorporating continuous wavelet transform-based pattern matching," Bioinformatics, vol. 22, no. 17, pp. 2059-2065, 2006.

[31] A. Wee, D. B. Grayden, Y. Zhu, K. Petkovic-Duran, and D. Smith, "A continuous wavelet transform algorithm for peak detection," Electrophoresis, vol. 29, no. 20, pp. 4215-4225, 2008.

[32] H. Golnabi and A. Asadpour, "Design and application of industrial machine vision systems," Robotics and ComputerIntegrated Manufacturing, vol. 23, no. 6, pp. 630-637, 2007.

[33] S. Lu, Real-time vertical track deflection measurement system, The University of Nebraska-Lincoln, 2008.

[34] Z. Zhang, "A flexible new technique for camera calibration," IEEE Transactions on Pattern Analysis and Machine Intelligence, vol. 22, no. 11, pp. 1330-1334, 2000.

[35] J. Weng, P. Cohen, and M. Herniou, "Camera calibration with distortion models and accuracy evaluation," IEEE Transactions on Pattern Analysis \& Machine Intelligence, vol. 14, no. 10, pp. 965-980, 1992.

[36] J. Heikkila and O. Silven, "A four-step camera calibration procedure with implicit image correction," in CVPR, Citeseer, 1997.

[37] G. Genta, P. Minetola, and G. Barbato, "Calibration procedure for a laser triangulation scanner with uncertainty evaluation," Optics and Lasers in Engineering, vol. 86, pp. 11-19, 2016.

[38] Y. Li, M. Kästner, and E. Reithmeier, "Triangulation-based edge measurement using polyview optics," Optics and Lasers in Engineering, vol. 103, pp. 71-76, 2018.

[39] B. A. Abu-Nabah, A. O. ElSoussi, and A. E. K. Al Alami, "Simple laser vision sensor calibration for surface profiling applications," Optics and Lasers in Engineering, vol. 84, pp. 51-61, 2016.

[40] M. Torabi, S. M. Mousavi G, and D. Younesian, "A new flexible laser beam profiler for the inspection of train wheels," Proceedings of the Institution of Mechanical Engineers, Part F: Journal of Rail and Rapid Transit, vol. 235, no. 2, pp. 215225, 2021.

[41] B. A. Chekh, G. Kortaberria, and O. Gonzalo, "Extrinsic calibration and kinematic modelling of a laser line triangulation sensor integrated in an intelligent fixture with 3 degrees of freedom," Precision Engineering, vol. 60, pp. 235-245, 2019. 
[42] J.-Y. Bouguet, “Camera calibration toolbox for matlab," 2012, http://www.vision.caltech.edu/bouguetj/calibdoc, 2013.

[43] J. Y. Bouguet and P. Perona, Camera Calibration from Points and Lines in Dual-Space Geometry, In Proceedings 5th European Conference on Computer Vision, Freiburg, Germany, 1998.

[44] H.-Y. Feng, Y. Liu, and F. Xi, "Analysis of digitizing errors of a laser scanning system,” Precision Engineering, vol. 25, no. 3, pp. 185-191, 2001. 